\title{
miR-145 suppresses ovarian cancer progression via modulation of cell growth and invasion by targeting CCND2 and E2F3
}

\author{
MINHUI HUA $^{1 *}$, YONGWEI QIN ${ }^{2 *}$, MEIHONG SHENG $^{3}$, XIAOPENG CUI ${ }^{4}$, \\ WEIGUAN CHEN $^{1}$, JIANXIN ZHONG ${ }^{1}$, JUNMING YAN ${ }^{2}$ and YAN CHEN ${ }^{2}$ \\ ${ }^{1}$ Department of Obstetrics and Gynecology, Affiliated Hospital of Nantong University; \\ ${ }^{2}$ Department of Pathogen Biology, Medical College, Nantong University; \\ ${ }^{3}$ Department of Radiology, The Second Affiliated Hospital of Nantong University; \\ ${ }^{4}$ Department of General Surgery, Affiliated Hospital of Nantong University, Nantong, Jiangsu 226001, P.R. China
}

Received February 8, 2018; Accepted January 28, 2019

DOI: $10.3892 / \mathrm{mmr} .2019 .10004$

\begin{abstract}
MicroRNAs (miRNA/miRs) have been demonstrated to be critical post-transcriptional modulators of gene expression during tumorigenesis. Numerous miRNAs have been revealed to be downregulated in human epithelial ovarian cancer (EOC). In the present study, it was observed that the expression of miR-145 was decreased in EOC tissues and cell lines. Overexpression of miR-145 inhibited the proliferation, migration and invasion of EOC cells. The D-type cyclin 2, cyclin D2 (CCND2), and E2F transcription factor 3 (E2F3) were confirmed to be targets of miR-145. In addition, restoration of these 2 genes significantly reversed the tumor suppressive effects of miR-145. Collectively, the results indicated that miR-145 serves a critical role in suppressing the biological behavior of EOC cells by targeting CCND2 and E2F3. Therefore, miR-145 was suggested to be a potential miRNA-based therapeutic target in ovarian cancer.
\end{abstract}

\section{Introduction}

Epithelial ovarian cancer (EOC) is the most lethal malignancy among females, exhibiting highly aggressive behaviors and a lack of early symptoms, leading to a large number of mortalities yearly (1). Although novel technologies have enabled advances in detection and therapeutic methods, improved understanding about the molecular events underlying this highly fatal form of tumorigenesis is required.

Correspondence to: Mrs Minhui Hua, Department of Obstetrics and Gynecology, Affiliated Hospital of Nantong University, 20 Xisi Road, Nantong, Jiangsu 226001, P.R. China

E-mail: mean0327@hotmail.com

${ }^{*}$ Contributed equally

Key words: microRNA-145, cyclin D2, 2F transcription factor 3, ovarian cancer
MicroRNAs (miRNA/miRs) have been demonstrated to be associated with the regulation of gene expression. miRNAs are endogenous RNA molecules that destabilize the transcripts of target genes or interact with complementary sequences in the 3'-untranslated region (UTR) of the target transcripts to repress their translation (2). By inhibiting translation, they lead to the downregulation of gene expression and increased degradation of RNAs, negatively regulating gene expression that may be involved in the regulation of critical cellular processes including the cell cycle, cell differentiation and apoptosis $(3,4)$. Dysregulation of miRNA expression may lead to the potentially abnormal regulation of their target mRNAs, which has been identified to be associated with clinical outcome in specific cancer types. In ovarian cancer, overexpressed miRNAs include miR-200a, miR-141, miR-200c and miR-200b; by contrast, miR-199a, miR-140, miR-145 and miR-125b1 are downregulated. The abnormal expression of these small molecules may allow them to serve as oncogenes or tumor suppressor genes, depending on the targets that they regulate (5).

miR-145 may modulate tumor cell growth, apoptosis and survival by targeting friend leukemia integration 1 transcription factor (6), insulin receptor substrate 1 (7), myc proto-oncogene protein (c-Myc) (8) and DNA fragmentation factor subunit alpha (DFF45) (9), and affect tumor migration, invasion and metastasis by targeting mucin 1 (MUC1) (10), p70S6 kinase (11) and 7,8-dihydro-8-oxoguanine triphosphate (12). Downregulation of miR-145 has been detected in breast (13) and colon cancer (7,11), lung adenocarcinoma (12), hepatocellular carcinoma (14), bladder cancer (15), pituitary tumors $(16)$, and ovarian $(11,17,18)$ and gallbladder cancer $(19)$. Previously, the mechanism through which the downregulation of miR-145 modulates cell growth and invasion in ovarian cancer by modifying the expression of certain oncogenes has been revealed in a small number of studies $(20,21)$, but to the best of our knowledge, no studies have investigated its regulatory functions in association with the targeting of cyclins protein and/or transcription factor.

Cyclin D2 (CCND2) belongs to the D-type cyclin family, the members of which serve as the primary integral mediators associated with cell cycle progression in the extracellular 
signaling environment (22). CCND2 forms a complex with cyclin-dependent kinase (CDK)4 or CDK6 and functions as a regulatory subunit of the complex, the activity of which is required for G1/S transition in the cell cycle (23). Through its ability to shorten the G1 phase, CCND2 primarily participates in cell cycle progression, and has therefore been implicated in the induction of cancer progression (24). E2F transcription factor $3(\mathrm{E} 2 \mathrm{~F} 3)$ is a member of the E2F transcription factor family involved in regulating cell proliferation (25). As an oncogene, it serves as a transcriptional activator and regulates the G1/S transition of the cell cycle $(26,27)$. The regulation of E2F1/2/3 activity is achieved by formation of a complex with retinoblastoma protein $(\mathrm{Rb})$, which drives proliferation by promoting the expression of target genes involved in DNA synthesis $(28,29)$. In this way, E2F3 may serve an essential role in the development of various types of cancer. CCND2 and E2F3 are target genes of miR-145; however, there is a lack of previous data regarding their association with ovarian cancer.

In the present study, the association between miR-145 and human ovarian cancer was first assessed, and then whether miR-145 was associated with CCND2 and E2F3 was examined in ovarian cancer cell lines, in order to explore the molecular mechanisms by which miR-145 may mediate cancer cell growth and invasion. The results may offer mechanistic insight into how miR-145 mediates the suppression of ovarian cancer.

\section{Materials and methods}

Tissue specimens and cell culture. The present study was approved by the Ethics Committee of the Affiliated Hospital of Nantong University. Tumor tissues were collected from 20 patients with serous ovarian cancer and eight patients with clear cell ovarian cancer between January 2015 and September 2016 (age, 43-67; mean age, 53.89; standard deviation of the mean age, 6.64) in the Affiliated Hospital of Nantong University. The normal tissues were collected from 20 patients with simple ovarian cysts or adenomyosis undergoing oophorectomy surgery. Written informed consent was obtained from all enrolled subjects.

Normal human ovarian surface epithelial cells (HOSEpiC; ScienCell Research Laboratories, Inc., Carlsbad, CA, USA) were cultured in RPMI-1640 medium, (Gibco; Thermo Fisher Scientific, Inc., Waltham, MA, USA) 293 cells (American Type Culture Collection, Manassas, VA, USA) were cultured in Dulbecco's modified Eagle's medium (DMEM; Gibco; Thermo Fisher Scientific, Inc.) medium, whereas ovarian cancer ES-2 and SKOV3 cells (American Type Culture Collection (Manassas, VA, USA) were cultured in McCoy's 5a Medium (HyClone; GE Healthcare Life Sciences, Logan, UT, USA). All media were supplemented with $10 \%$ fetal bovine serum (FBS; Gibco; Thermo Fisher Scientific, Inc.). The cells were maintained in a humidified incubator at $37^{\circ} \mathrm{C}$ with $5 \%$ $\mathrm{CO}_{2}$.

$R N A$ extraction and reverse transcription quantitative polymerase chain reaction ( $R T-q P C R)$. Total RNA (including miRNA) was extracted from cells and tissues using TRIzol ${ }^{\mathbb{}}$ (Thermo Fisher Scientific, Inc.) according to the manufacturer's protocol. PCR was performed with SYBR Green PCR Master Mix (Applied Biosystems; Thermo Fisher Scientific,
Inc.) according to the manufacturer's protocol. For miRNA detection, 10 ng RNA was reverse transcribed using a miRNA RT kit (cat. no. 4366596; Thermo Fisher Scientific, Inc.), and the miR-145 primers used were as follows: Stem-loop primer, 5'-GTCGTATCCAGTGCAGGGTCCGAGGTATTC GCACTGGATACGACAGGGAT-3'; PCR forward (F) primer, 5'-GTCCAGTTTTCCCAGGAATCC-3', PCR reverse (R) 5'-CAGTGCAGGGTCCGAGGTAT-3'. The reaction conditions were as follows: $95^{\circ} \mathrm{C}$ for $5 \mathrm{~min}$, followed by 40 cycles of amplification at $95^{\circ} \mathrm{C}$ for $30 \mathrm{sec}, 57^{\circ} \mathrm{C}$ for $30 \mathrm{sec}$ and $72^{\circ} \mathrm{C}$ for $30 \mathrm{sec}$. The primers for CCND2 were 5'-CAGCCGTCC ACTTCAGC-3' (F) and 5'-TGCCTTTGGGTCTTCC-3' (R). The primers for E2F3 were 5'-CCCTAAACCCGCTTCC-3' (F) and 5'-GTTCACAAACGGTCCTTCTA-3' (R). Conditions for amplifying mRNAs were as follows: Initial denaturation of $95^{\circ} \mathrm{C}$ for $30 \mathrm{sec}$, followed by 40 cycles of $95^{\circ} \mathrm{C}$ (denaturation) for $5 \mathrm{sec}$ and $60^{\circ} \mathrm{C}$ for $30 \mathrm{sec}$ (annealing and elongation, two-step PCR). The expression of miR-145 was normalized to that of U6 small nuclear B non-coding RNA (RNU6B), while the levels of CCND2 and E2F3 were normalized to those of GAPDH, using the $2^{-\Delta \Delta C q}$ method (30). The primers for GAPDH were 5'-AGGTGGTCTCCTCTGACTTCAA-3' (F) and 5'-TTCGTTGTCATACCAGGAAATG-3' (R). The RNU6B RT primer (stem-loop primer) was 5'-AACGCTTCA CGAATTTGCGT-3', and the PCR primers were 5'-CTCGCT TCGGCAGCACA-3' (F) and 5'-AACGCTTCACGAATT TGCGT-3' (R).

Plasmid construction and transfection. Overexpression of miR-145 was established using human miR-145 mimics (5'-GUCCAGUUUUCCCAGGAAUCCCU-3') and the corresponding mimic controls (5'-UUCUCCGAACGU GUCACGU-3'), obtained from Shanghai GenePharma Co., Ltd., (Shanghai, China). The CCND2 overexpression vector primers were 5'-GGGGTACCATGGAGCTGCTGT GCCACGAGGTGG-3' and 5'-GCTCTAGATCACAGG TCGATATCCCGCACGTCT-3'. The E2F3 overexpression vector primers were 5'-CGGGATCCAGACTTGGAAACT CCGACTG-3' and 5'-CGGAATTCTTGGAGGAAGAAG GTAGGAA-3'. The primers were purchased from Sangon Biotech Co., Ltd. (Shanghai, China). The DNA was extracted from SKOV3 cells. The PCR reaction conditions were as follows: Initial denaturation at $95^{\circ} \mathrm{C}$ for $5 \mathrm{~min}$, followed by 40 cycles of amplification at $95^{\circ} \mathrm{C}$ for $30 \mathrm{sec}, 55^{\circ} \mathrm{C}$ for $30 \mathrm{sec}$ and $72^{\circ} \mathrm{C}$ for $60 \mathrm{sec}$ using Takara LA PCR kit Ver.2.1 (Takara Biotechnology Co., Ltd., Dalian, China). The PCR products were inserted into a pcDNA3.1 plasmid (Shanghai GenePharma Co., Ltd.). The thermocycling conditions were as follows: 40 cycles of denaturation at $94^{\circ} \mathrm{C}$ for $30 \mathrm{sec}$, annealing at $60^{\circ} \mathrm{C}$ for $15 \mathrm{sec}$ and extension at $72^{\circ} \mathrm{C}$ for $45 \mathrm{sec}$ using Takara LA PCR kit (Takara Biotechnology Co., Ltd.). Cell transfection was performed using Lipofectamine ${ }^{\circledR}$ 2000 (Invitrogen; Thermo Fisher Scientific, Inc.) for $48 \mathrm{~h}$ according to the manufacturer's protocol. In total, miRNA at a concentration of $50 \mathrm{nM}$ was transfected with $1 \mu \mathrm{g}$ expressing plasmids using $1.5 \mu 1$ Lipofectamine ${ }^{\circledR} 2000$ in each well.

3'-UTR luciferase reporter assays. The 3'-UTRs of CCND2 (forward primer, 5'-GGAGTTCTTGGGAATCTTG-3' and 
reverse primer, 5'-CCCTTTAGTGGGAGGTAA-3') and E2F3 (forward primer, 5'-GCCAGTTTACTCCAGGTA-3' and reverse primer, 5'-AACAATCTAGCCAGGTGA-3') were amplified by PCR as above-mentioned from a human SKOV3 cell cDNA library and cloned downstream of the Renilla luciferase coding sequence, between the Xho I and Not I sites, of the psiCHECK2 luciferase reporter vector (Promega Corporation, Madison, WI, USA). The miR-145 target site in the CCND2 and E2F3 3'UTRs was mutated by altering the 3-nt miR-145 seed match sequence using the QuikChange Site-Directed Mutagenesis kit (Stratagene; Agilent Technologies, Inc., Santa Clara, CA, USA), according to manufacturer's protocol. The aforementioned 293 cells were cotransfected with $100 \mathrm{ng}$ psiCHECK2-CCND2 3'UTR or psiCHECK2-E2F3 3'UTR, or psiCHECK2-CCND2 3'UTR-Mutant (Mut) or psiCHECK2-E2F3 3'UTR-Mut luciferase plasmid, and the miR-145 mimics or miR-145-negative controls (NC) using Lipofectamine ${ }^{\circledR} 2000$. After 24 h, luciferase activity was measured using the Dual-Glo Luciferase Reporter Assay System (Promega Corporation) according to the manufacturer's instructions. Data were normalized for transfection efficiency by comparison with Renilla luciferase activity.

Bioinformatics prediction. CCND2 and E2F3 were identified as potential targets of miR-145 using the bioinformatics software TargetScan(version 7.0; http://www.targetscan.org/cgibin/targetscan/vert_71/targetscan.cgi?species=Human\&gid= $\&$ mir_sc=\&mir_c=\&mir_nc=\&mir_vnc $=\& \operatorname{mirg}=\operatorname{miR}-145)$.

Cell proliferation assay. A total of $24 \mathrm{~h}$ after transfection, target cells were seeded into 96 -well plates at a density of 2,000 cells/well and cultured for 0, 24, 48, 72 and $96 \mathrm{~h}$. Cell proliferation was detected by Cell Counting Kit-8 (CCK-8) assay according to the manufacturer's protocol (LakePharma, Inc., San Carlos, CA, USA). Absorbance values were detected at a wavelength of $450 \mathrm{~nm}$. A total of 3 wells were measured for cell viability for each group.

Transwell invasion assay. Transwell chambers (EMD Millipore, Billerica, MA, USA) were used to examine the migration and invasion of cells. Filters for the invasion assay were precoated at $37^{\circ} \mathrm{C}$ for $2 \mathrm{~h}$ with Matrigel (BD Biosciences, Franklin Lakes, NJ, USA). A total of $\sim 5 \times 10^{4}$ cells were added to the upper Transwell chamber and cultured in serum-free DMEM. The lower compartment of the Transwell chamber was filled with DMEM containing 10\% FBS. Cells remaining on the lower surface were fixed for $30 \mathrm{~min}$ at room temperature with $4 \%$ formaldehyde (Sangon Biotech Co., Ltd.) and stained with $0.5 \%$ crystal violet (Sangon Biotech Co., Ltd.) at room temperature for $60 \mathrm{~min}$. A total of four randomly-selected microscope (light microscope; magnification, $\mathrm{x} 20$ ) fields were counted for each group.

Cell cycle analysis. The cells were transfected with miR-145 mimics and miR-145 NC, and CCND2, E2F3 and CCND2 + E2F3 overexpression plasmids for $48 \mathrm{~h}$, following which the cells were collected and washed with PBS. Then, the cells were washed with $1 \%$ bovine serum albumin (Sigma-Aldrich; Merck KGaA, Darmstadt, Germany) and fixed in 95\% ice-cold ethanol containing $0.5 \%$ Tween- 20 for $2 \mathrm{~h}$ at $-20^{\circ} \mathrm{C}$. Following fixation, cells $\left(1 \times 10^{6}\right)$ were washed with cold $1 \%$ BSA and stained with $10 \mu \mathrm{g} / \mathrm{ml}$ propidium iodide (Sigma-Aldrich; Merck KGaA) in a solution containing $100 \mu \mathrm{g} / \mathrm{ml}$ RNase in PBS for $30 \mathrm{~min}$ at room temperature in the dark. The cells were analyzed using a flow cytometer (BD FACSCalibur ${ }^{\mathrm{TM}}$, BD Biosciences, San Jose, CA, USA). BD CellQuest Pro software (version, 5.1; BD Biosciences) was used to analyze the flow cytometry data.

Wound healing assay. Cell migration was assessed by a wound-healing assay. Cells $\left(2 \times 10^{6}\right)$ were seeded in 6-well plates and transfected the following day. At $12 \mathrm{~h}$ after transfection, cells were incubated at $37^{\circ} \mathrm{C}$ for $24 \mathrm{~h}$, and $95 \%$ confluent cells were used for wound healing assay. Wounds were made using a pipette tip. The wounded areas were observed and imaged under a light microscope (200x). The migration distances were imaged at 0 and $24 \mathrm{~h}$ after scratching, and the change in cell migration was determined by comparing the difference in the wounded area in at least 4 fields. Experiments were performed in triplicate and repeated at least 3 times.

Western blot analysis. Cell lysates were prepared using a lysis buffer (Beyotime Institute of Biotechnology, Haimen, China). The protein concentration in each sample was assessed using a spectrophotometer (Nanodrop 1000; Thermo Fisher Scientific, Inc.). A total of $80 \mu \mathrm{g}$ protein was loaded in each lane. Proteins were separated by $10 \%$ SDS-PAGE, and transferred to polyvinylidene difluoride membranes (EMD Millipore). Following blocking using 5\% non-fat milk for $2 \mathrm{~h}$ at room temperature, the membranes were incubated with anti-CCND2 monoclonal antibody (cat. no. SC-56305; 1:500; Santa Cruz Biotechnology, Inc., Dallas, TX, USA), anti-E2F3 polyclonal antibody (cat.no.SC-879; 1:500; Santa CruzBiotechnology,Inc.), anti-B cell lymphoma 2 (Bcl-2; cat. no. 4223; 1:1,000; Cell Signaling Technology, Inc., Danvers, MA, USA), anti-Bcl-2-associated $\mathrm{X}$ protein (Bax; cat. no. 5023; 1:1,000; Cell Signaling Technology, Inc.) and anti- $\beta$-actin (cat. no. 4970; 1:2,000; Cell Signaling Technology, Inc.) overnight at $4^{\circ} \mathrm{C}$, which was followed by incubation with anti-mouse (cat. no. 7076; 1:4,000; Cell Signaling Technology, Inc.) or anti-rabbit (cat. no. 7074; 1:5,000; Cell Signaling Technology, Inc.) horseradish peroxidase-conjugated immunoglobulin $\mathrm{G}$ at $4^{\circ} \mathrm{C}$ for $2 \mathrm{~h}$. The blots were developed using an enhanced chemiluminescent system (Amersham; GE Healthcare, Chicago, IL, USA), and the density of bands was detected using ImageJ 1.42q software (National Institutes of Health, Bethesda, MD, USA).

Statistical analysis. Data were presented as mean \pm standard error of the mean. Statistical analysis was performed using SPSS 11.0 for Windows (SPSS, Inc., Chicago, IL, USA) and GraphPad Prism 7.0 for Windows (GraphPad Software Inc., La Jolla, CA, USA). The difference between two groups was analyzed by Student's t-test. One-way analysis of variance followed by Tukey's post hoc test was used to compare multiple groups. $\mathrm{P}<0.05$ was considered to indicate a statistically significant difference. All data are presented as the mean \pm standard error of the mean. 

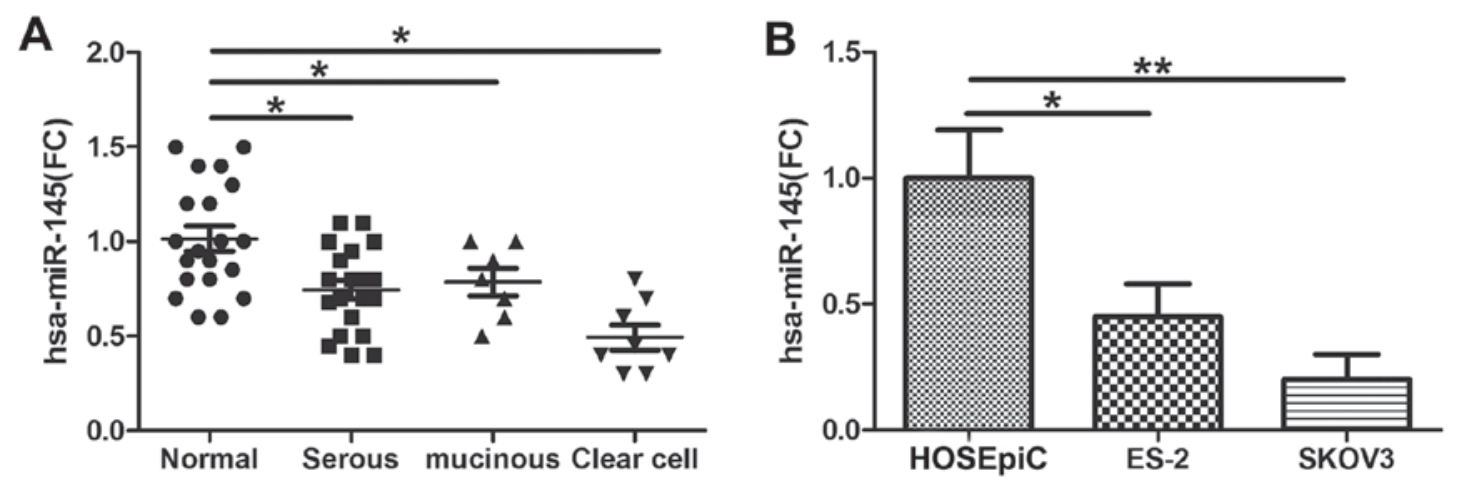

Figure 1. Expression of miR-145 was determined by RT-qPCR in normal ovarian tissues and tissues from different types of ovarian cancer. (A) The expression of miR-145 was determined by RT-qPCR in ovarian cancer cells lines. (B) Significant expression of miR-145 was demonstrated in ovarian tissue and cancer cells by Friedman two-way analysis of variance. ${ }^{*} \mathrm{P}<0.05$ and ${ }^{* *} \mathrm{P}<0.01$. miR, microRNA; RT-qPCR, reverse transcription polymerase chain reaction; serous, serous ovarian cancer; clear cell, clear cell carcinoma; FC, fold change; HOSEpiC, human ovarian surface epithelial cells

\section{Results}

miR-145 is downregulated in human ovarian cancer tissues and cell lines. The expression level of miR-145 was examined via $\mathrm{qPCR}$ in tumor tissues obtained from patients with ovarian cancer (from 20 patients with serous ovarian cancer and 8 with clear cell ovarian cancer). The data indicated that miR-145 expression was decreased in serous ovarian cancer and clear cell ovarian cancer tissues compared with control tissues (Fig. 1A). In concordance with the results in the cancer tissues, miR-145 was significantly downregulated in ovarian cancer ES-2 and SKOV3 cell lines compared with HOSEpiC cells (Fig. 1B).

miR-145 suppresses the proliferation and invasion of ovarian cancer cells. To investigate the functions of miR-145 in ovarian cancer cells, miR-145 mimics and mimic NC were transfected into ES-2 and SKOV3 cells. Then, qPCR was performed to measure the expression of miR-145. The expression of miR-145 was notably increased in miR-145 mimic-transfected cells (Fig. 2A). In a CCK-8 assay, the overexpression of miR-145 significantly inhibited the proliferation of ovarian cancer cells at $96 \mathrm{~h}$ (Fig. 2B). In addition, wound healing (Fig. 2C) and invasion (Fig. 2D) assays also indicated that miR-145 overexpression markedly suppressed the migratory and invasive capabilities, which are characteristic metastatic behaviours, of ovarian cancer cells. Bax and Bcl-2 are members of a family of cytoplasmic proteins that regulate apoptosis. To investigate the potential of miR-145 to induce apoptosis, the levels of pro-apoptotic protein Bax and the anti-apoptotic protein $\mathrm{Bcl}-2$ were measured in miR-145-transfected or corresponding control-transfected SKOV3 and ES-2 cells. Compared with those cells transfected with miR-145 NC, the miR-145-transfected cells exhibited a decrease in Bcl-2 expression and an increase in Bax expression. These results suggested that miR-145 promoted the apoptosis of SKOV3 and ES-2 cells (Fig. 2E).

CCND2 and E2F3 are targets of miR-145 in ovarian cancer cells. CCND2 and E2F3 were identified as potential targets of miR-145 using the bioinformatics software TargetScan.
To validate this prediction, luciferase candidate genes were constructed with the 3'-UTR of each target downstream of the reporter (Fig. 3A). The luciferase activity assays identified that miR-145 mimics significantly inhibited luciferase activity when cells were co-transfected with luciferase plasmid containing the wild type (WT) but not the Mut 3'-UTR of CCND2 or E2F3 (Fig. 3B). RT-qPCR assays also demonstrated that the relative mRNA expression levels of CCND2 and E2F2 were downregulated following transfection with miR-145 in SKOV3 cells (Fig. 3C). Furthermore, CCND2 and E2F3 expression was detected in ovarian cancer tissues. The results of the western blot analysis indicated that the protein levels of CCND2 and E2F3 were increased in ovarian cancer tissues compared with the normal tissues (Fig. 3D). Additionally, in SKOV3 cells, overexpression of miR-145 significantly suppressed CCND2 and E2F3 protein levels (Fig. 3E and F). Taken together, these data verified a direct interaction between miR-145 and the two target genes CCND2 and E2F3.

Restoration of CCND2 and E2F3 reverses the effects of $m i R-145$. CCND2 or E2F3, alone or together, were not transfected into the ovarian cancer cell line in the absence of miR-145 to clarify the role of CCND2 and E2F3 in tumorigenesis, as it has been confirmed in a previous study (31). Whether CCND2 and E2F3 were associated with the function of miR-145 was additionally investigated. A total of 2 cell lines were transfected with CCND2 and E2F3 overexpression plasmids, and expression patterns of the proteins were examined by western blot analysis. The protein expression levels of CCND2 and E2F3 were significantly increased in the cells transfected with the target gene overexpression plasmids (Fig. 4A). Subsequently, the cells were co-transfected with miR-145 mimics or NC vector and the target genes overexpression plasmids. The effect of miR-145 and its target genes on cell proliferation was examined using the CCK-8 assay (Fig. 4B). It was observed that cell viability gradually increased over the indicated time points following transfection with CCND2 or E2F3 overexpressed plasmids. The effects of miR-145 and its target genes on cell cycle progression were also determined using flow cytometry, which revealed the percentages of ES-2 and SKOV3 cells in G1 phase to be increased 


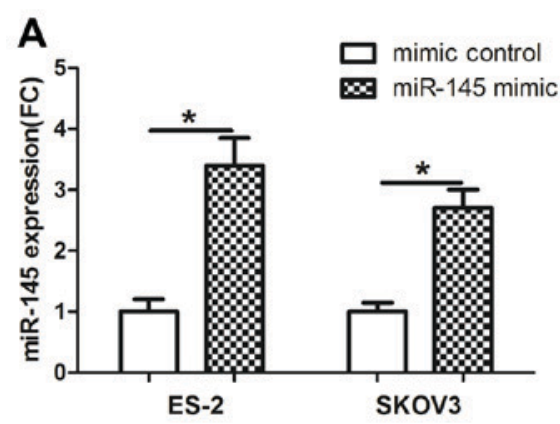

C

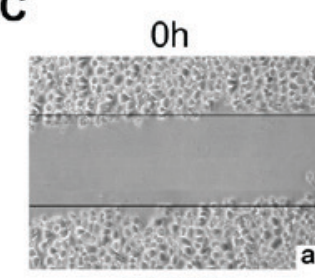

sKov3
B

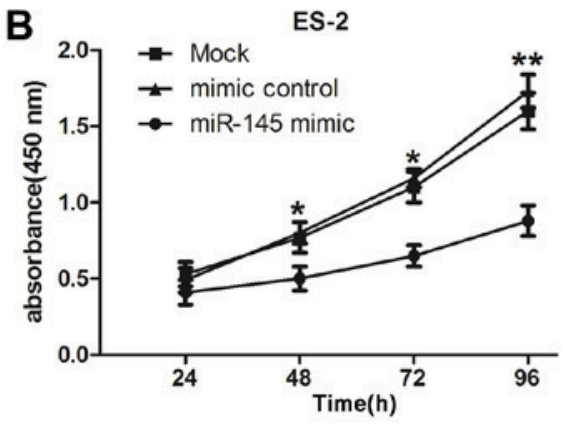

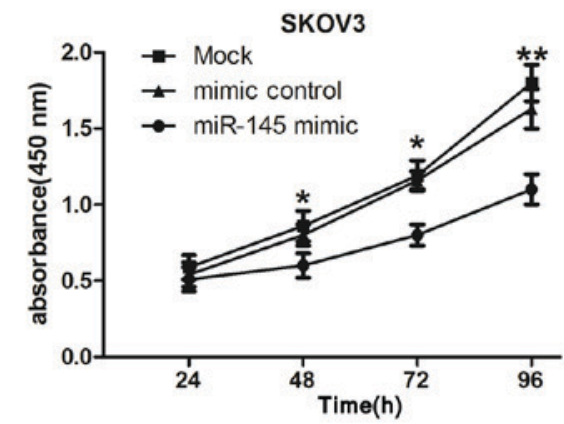

D

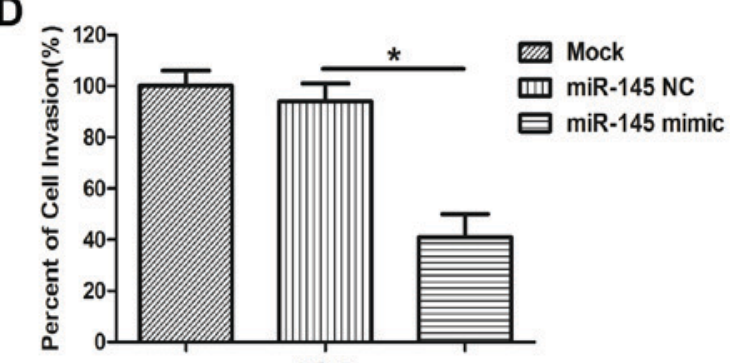

ES-2

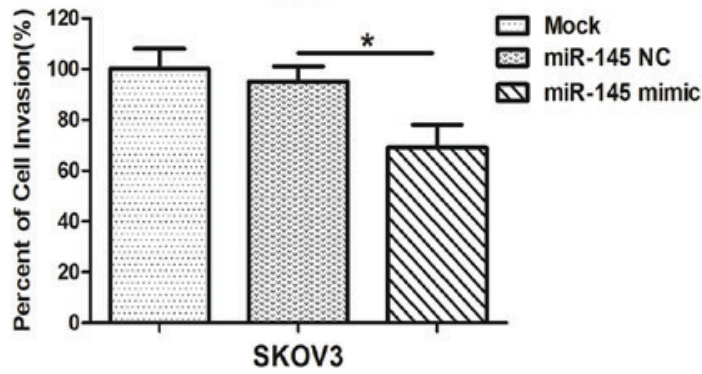

SKOV3

E

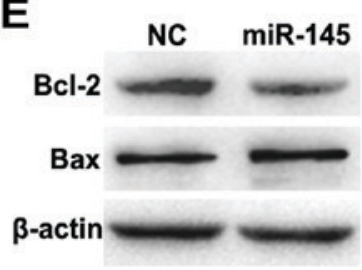

SKov3

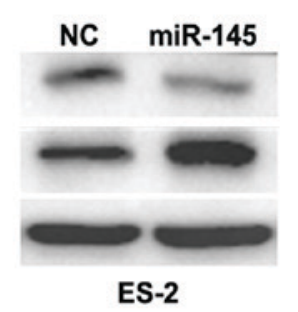

$24 \mathrm{~h}$

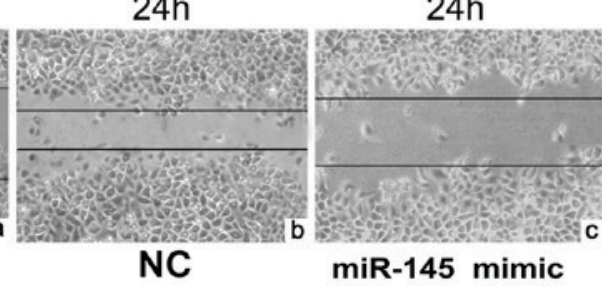

miR-145 mimic

Figure 2. miR-145 inhibits ovarian cancer cell proliferation, cell cycle, invasion and migration. (A) SKOV3 and ES-2 cells were transfected with miR-145 mimics or the control mimics, and the expression of miR-145 was determined by reverse transcription quantitative polymerase chain reaction. (B) Cell viability in cells transfected with miR-145 mimics or NC was measured by Cell Counting Kit-8 assay at 4 time points $(0,24,48,72$ and 96 h). UV-visible absorbance was measured at $450 \mathrm{~nm}$. ${ }^{*} \mathrm{P}<0.05$ and ${ }^{* *} \mathrm{P}<0.01$ for mimic control and miR-145 mimic groups vs. mock group. (C) Wound healing assay was performed at $24 \mathrm{~h}$ post-transfection (magnification, 200x). (C-a) SKOV3 cells at $0 \mathrm{~h}$. Following $24 \mathrm{~h}$, the migration ability of SKOV3 cells transfected with (C-b) miR-NC significantly increased compared with (C-c) cells transfected with miR-145. The results indicated that overexpression of miR-145 inhibits cell migration. (D) Invasion assays of ovarian cancer cells transfected with miR-145 or the control mimics. (E) The expression levels of Bcl-2 and Bax were detected in SKOV3 and ES-2 cells using western blot analysis. Experiments were performed in triplicate. " $\mathrm{P}<0.05$ and ${ }^{* *} \mathrm{P}<0.01$. miR, microRNA; NC, negative control; FC, fold change.

following transfection with miR-145, compared with those of the controls, providing evidence that upregulated miR-145 induced G1 cell cycle arrest in ovarian cancer cells. Similarly, CCND2 and E2F3 significantly increased the populations of SKOV3 and ES-2 cells in S phase, and therefore appeared to promote entry into the $\mathrm{S}$ phase, thereby increasing proliferation (Fig. 4C and D). In addition, wound healing (Fig. 4E) and invasion (Fig. 4F) assays demonstrated that restoration of CCND2 or E2F3 significantly reversed the tumor suppressive effects of miR-145. Overall, these data suggest that miR-145 functions as a tumor suppressor at least in part by targeting CCND2 and E2F3.

\section{Discussion}

Increasing evidence has demonstrated that miRNAs are significantly involved in the differentiation, proliferation and apoptosis of various malignancies by interacting with the 3'-UTR of their target genes. miR-145 is located on chromosome 5 (5q32-33), an established fragile site in the human genome (32). It targets numerous oncogenes, which regulate cellular processes including the cell cycle, proliferation, apoptosis and invasion. Accordingly, decreased expression of miR-145 has been revealed in a number of types of cancer. However, limited studies have demonstrated the downregulation of miR-145, as a tumor suppressor, in human ovarian cancer, which has been suggested as a potential diagnostic marker or as a target for sensitizing ovarian cancer cells to chemotherapy $(33,34)$. The results from the present study suggested that miR-145 was downregulated in 3 different pathological types of EOC, and furthermore that its expression was decreased in the ovarian cancer cell lines SKOV3 and ES-2. miR-145 mimics were subsequently transfected into the cell lines, and the increased expression of miR-145 was confirmed by qPCR. In the following experiments, it was identified that the percentage of cells in G1 phase increased, while cell proliferation, migration and invasion were suppressed. These results indicate that miR-145 may exert its inhibitory effects on its target oncogenes and thereby mediate cell cycle regulation in ovarian cancer cells.

At present, a number of oncogenes have been identified as targets of miR-145. Previous studies have confirmed that 
A

CCND2 3' UTR (Position 1855-1861)

WT 3'UTR ...AUAUGAGUUCUUCGUACUGGAAA...
Hsa-miR-145 UCCCUAAGGACCCUUUUGACCUG
Mut 3'UTR ...AUAUGAGUUCUUCGUAGUCCAGA...

E2F3 3' UTR (Position 2029-2035)

WT 3'UTR $\quad 5$ ' ...UUGUGUUAAGUGCCUACUGGAAA...

Hsa-miR-145 3' UCCCUAAGGACCCUUUUGACCUG

Mut 3'UTR 5 ' ...UUGUGUUAAGUGCCUAGUCCAAA...

C
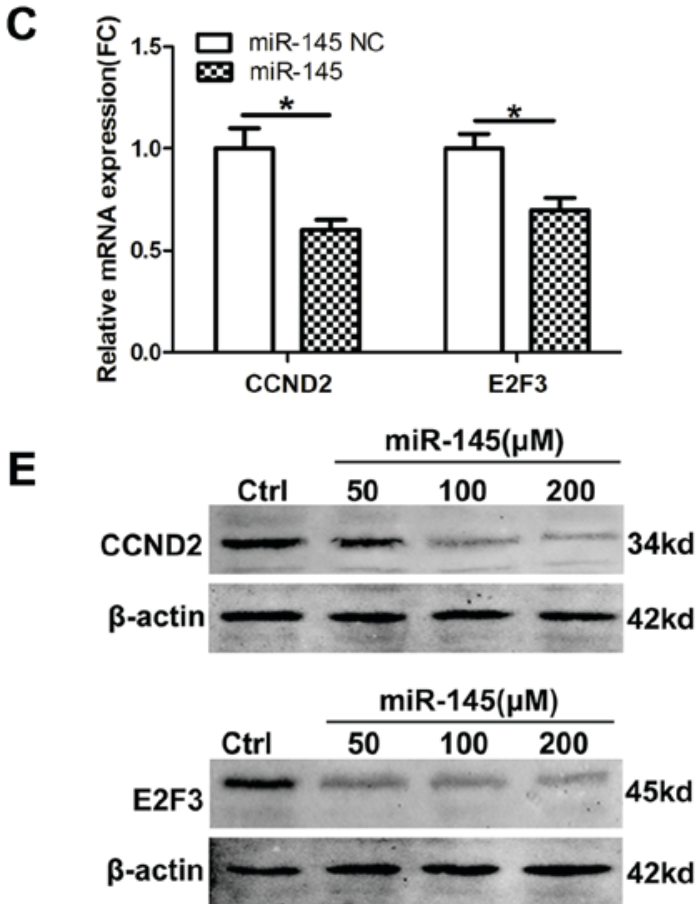

B
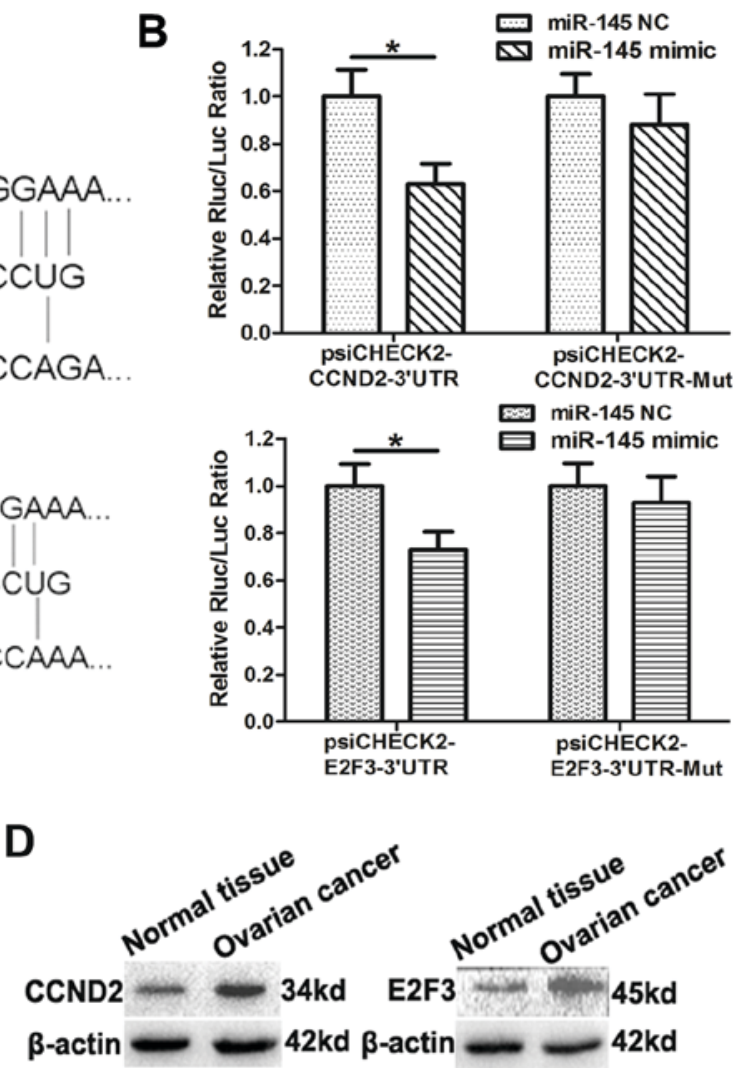

$\mathbf{F}$

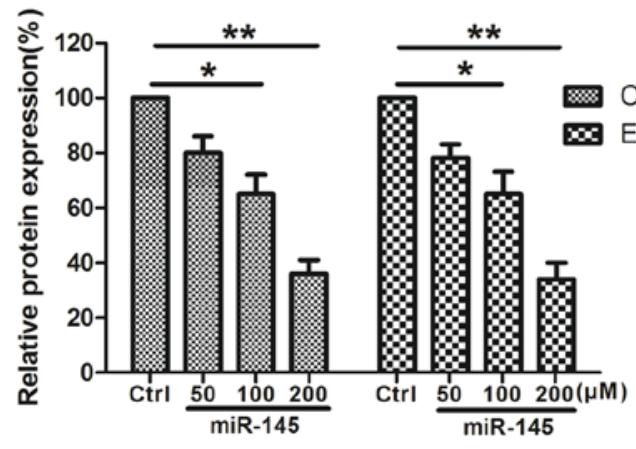

Figure 3. CCND2 and E2F3 are the targets of miR-145 in ovarian cancer cells. (A) The schematic construction of WT and Mut 3'-UTR of CCND2 and E2F3. (B) SKOV3 cells were co-transfected with WT or Mut 3'-UTR of CCND2 and E2F3, followed by transfection with the miR-145 or NC mimics. The luciferase activities were examined $48 \mathrm{~h}$ after transfection. (C) SKOV3 cells were transfected with miR-145 or the control mimics, and CCND2 and E2F3 mRNA were detected by qPCR. (D) The expression levels of CCND2 and E2F3 were detected in ovarian cancer tissues and normal ovarian tissues using western blot analysis. (E) The protein level of CCND2 and E2F3 were detected in SKOV3 cells transfected with miR-145 or the NC mimics. (F) Densitometric evaluation of band intensities. Experiments were performed in triplicate. " $\mathrm{P}<0.05$ and ${ }^{* *} \mathrm{P}<0.01$ vs. control. CCND2, cyclin D2; E2F3, E2F transcription factor 3; miR, microRNA; WT, wild type, Mut, mutant; UTR, untranslated region; NC/ctrl, negative control; FC, fold change.

miR-145 may suppress cell proliferation by targeting growth factor-associated genes including insulin receptor substrate 1 , insulin like growth factor receptor and epidermal growth factor receptor (EGFR) $(14,35,36)$. It may also inhibit DFF45, protein phosphatase 3 catalytic subunit alpha, core-binding factor subunit beta, clathrin interactor 1 and c-Myc, promoting cell apoptosis and interrupting the cell cycle. In addition, certain oncogenes that are involved in cell invasion and metastasis, including neural precursor cell expressed, developmentally down-regulated 9, fascin actin-bundling protein 1, MUC1, cell surface associated and sex-determining region box 9, may also be regulated by miR-145 (10,37-40). The identification of an increasing number of target genes indicates that miR-145 may be involved in a complicated regulatory network. In the present study, it was confirmed that CCND2 and E2F3 were direct targets of miR-145 by a luciferase activity assay. D-type cyclins (D1, D2 and D3) are considered to be potentially oncogenic proteins, as they serve critical roles in regulating cell cycle progression through the G1 phase to S phase. D-type cyclins bind to and activate CDK4/CDK6, which induces cell cycling $(40,41)$. The cyclin D-CDK4/CDK6 complex may inactivate $\mathrm{Rb}$ during early $\mathrm{G} 1$ phase by progressive multi-phosphorylation, resulting in the upregulation of transcription factor E2F. E2F may then target genes that promote 

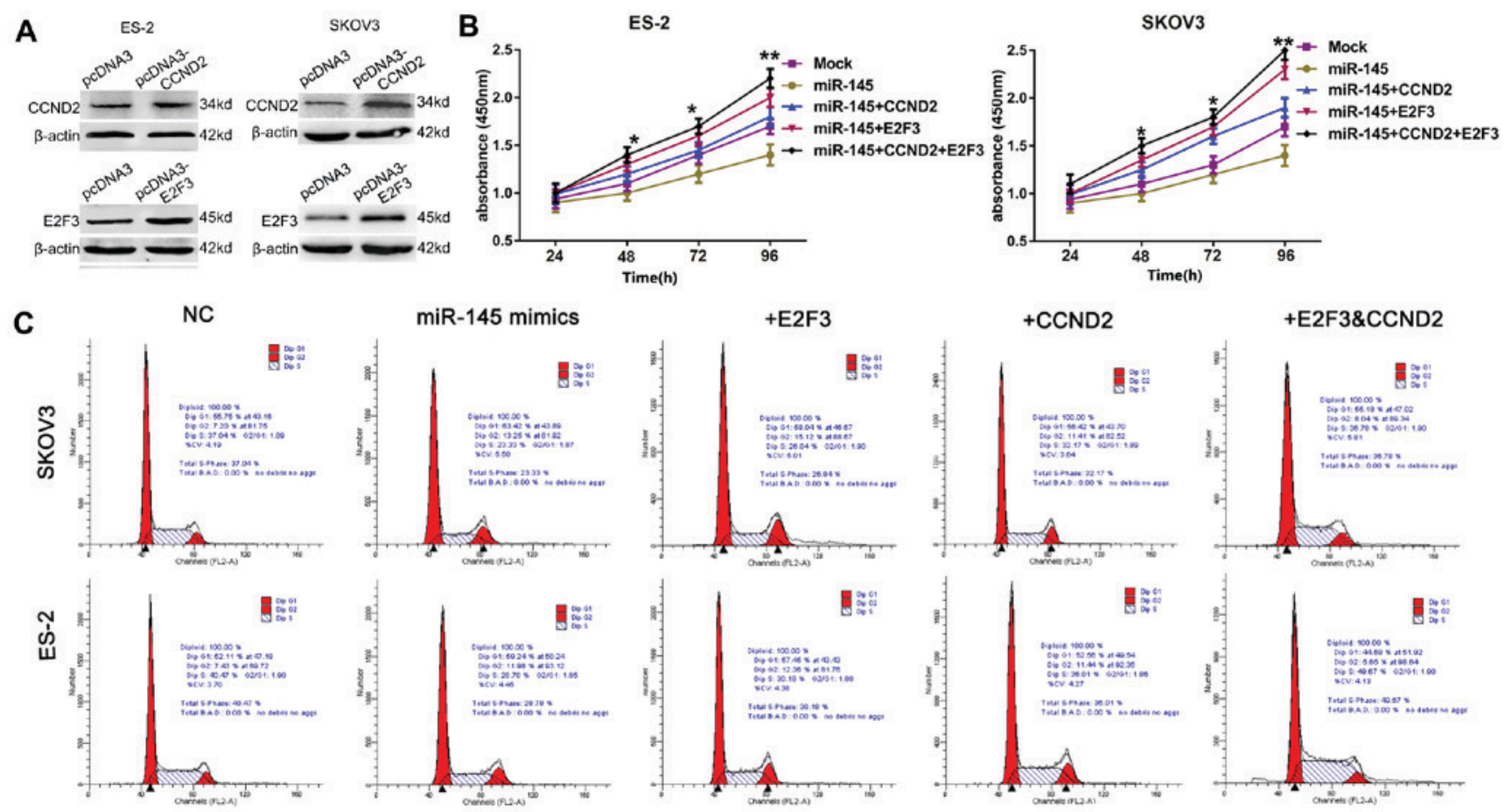

D
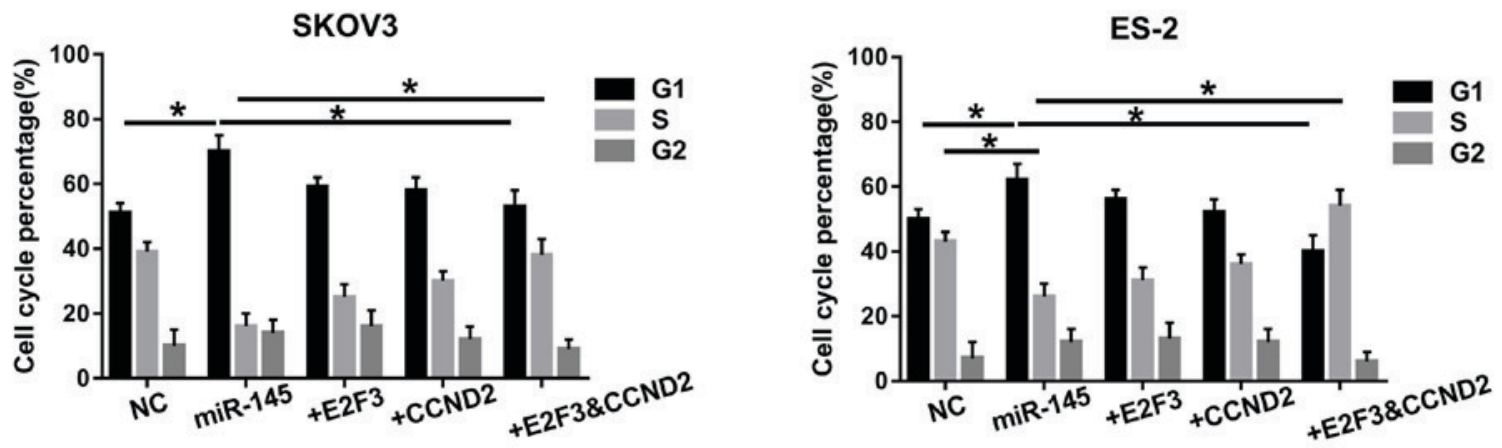

E

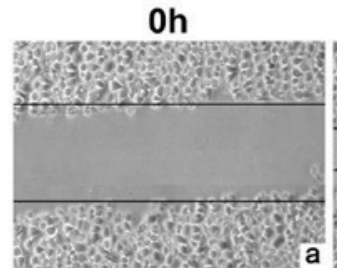

SKOV3
$24 \mathrm{~h}$

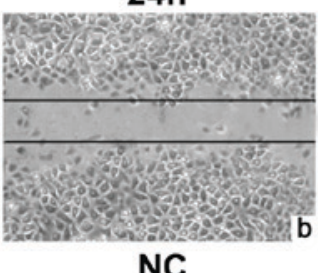

NC

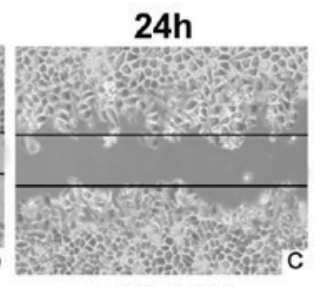

miR-145

+E2F3+CCND2

\section{$\mathbf{F}$}

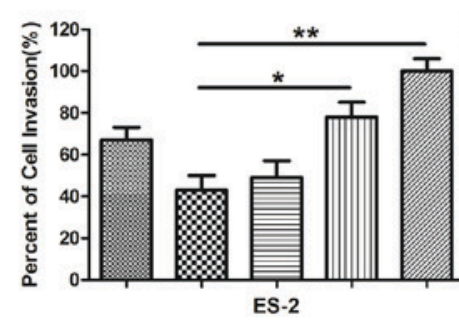

․․․ Mock

miR-145

曰 miR-145+CCND2

血 miR-145+E2F3

miR-145+CCND2+E2F3

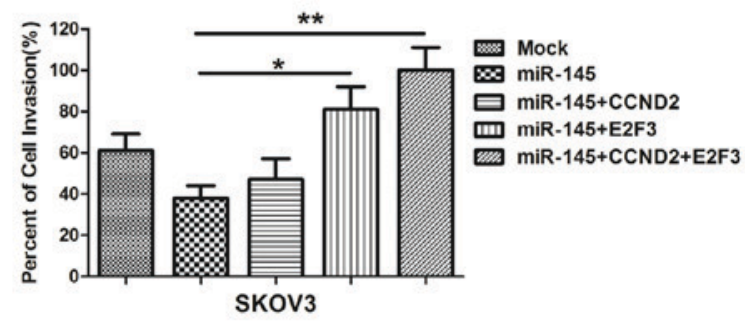

Figure 4. Restoration of CCND2 and E2F3 expression reverses the effects of miR-145. (A) SKOV3 and ES-2 cells were transfected with CCND2 and E2F3 overexpression plasmids, and the expression was then examined by western blot analysis. The 2 cells lines were also cotransfected with miR-145 mimic + CCND2 and E2F3 overexpression plasmids or the control vector. (B) Cell viability was measured by Cell Counting Kit-8 assay. (C) SKOV3 and ES-2 cells were stained with propidium iodide and subjected to flow cytometry to determine the distribution of cells at each phase of the cell cycle, and were compared with the NC group. The proportion of G1 phase decreased in the cells transfected with CCND2 and E2F3 overexpression plasmids. (D) Quantification of cell cycle distribution results presented as the mean \pm standard error of the mean of 3 independent experiments. ${ }^{*} \mathrm{P}<0.05$ and ${ }^{* * *} \mathrm{P}<0.01$. (E) Wound healing assay was performed at $24 \mathrm{~h}$ post-transfection (magnification, 200x). (E-a) SKOV3 cells at $0 \mathrm{~h}$. Following $24 \mathrm{~h}$, the migration of SKOV3 cells transfected with (E-b) NC was not significantly altered compared with cells transfected with (E-c) miR-145, E2F3 and CCND2. The results indicated that the effects of miR-145 were reversed by CCND2 and E2F3. (F) Invasion assays were performed. Experiments were performed in triplicate. " $\mathrm{P}<0.05$ and ${ }^{* * *} \mathrm{P}<0.01$ vs. the control. CCND2, cyclin D2; E2F3, E2F transcription factor 3; miR, microRNA; NC, negative control. 
cell cycle progression into the $\mathrm{S}$ phase (42), including cyclin E, cyclin A, CDK2 and cell cycle division $25(41,43)$. Certain studies have demonstrated that CCND2 and E2F3 are associated with tumorigenesis (31). Chang et al (44) revealed that CCND2 is involved in stimulating the proliferation, cell cycle progression, migration and invasion of ovarian cancer cells. In addition, E2F3 is critically involved in EGFR-mediated proliferation in ovarian cancer and is clinically relevant in ovarian cancer (45). Therefore, experiments to clarify the function of CCND2 and E2F3 on cell growth, migration and invasion were not repeated in the present study. The present study aimed to clarify whether the participation of CCND2 and E2F3 in cell growth, migration and invasion was mediated by miR-145.

The results indicated that the protein expression levels of CCND2 and E2F3 were downregulated by transfection with miR-145. Conversely, the restoration of CCND2 and E2F3 by transfection with CCND2 and E2F3 overexpression plasmids reversed the effects of miR-145. This restoration was observed in cells transfected with separate CCND2 and E2F3 overexpressed plasmids; the co-transfection of the 2 genes together additionally augmented the restoration. Collectively, the data from the present study confirmed the effect of miR-145 in targeting upstream (CCND2) and downstream (E2F3) molecules, which appeared to markedly affect the biological behavior of ovarian cancer cells. It was also observed that the effect of miR-145 was not only partially reversed, but abrogated compared with the mock control, and it was hypothesized that CCND2 and E2F3 may participate in other potential mechanisms, which led to this result. In summary, the present study demonstrated that miR-145 expression was decreased in serous ovarian cancer and clear cell ovarian cancer tissues, and in the corresponding ovarian cancer cell lines. Overexpression of miR-145 was able to suppress ovarian cancer cell proliferation, migration and invasion. Additionally, CCND2 and E2F3 were confirmed as targets of miR-145 in ovarian cancer cells, and restoration of these 2 genes partly reversed the tumor-suppressive effect of miR-145. The results of the present study suggested that miR-145 may function as a tumor-inhibiting factor by regulating the oncogenes CCND2 and E2F3 in ovarian cancer. An in vivo study will be conducted in future to verify these data. A mouse model of ovarian cancer has been established (unpublished data), in order to confirm the effect of miR-145 on the biological behavior of ovarian cancer by local injection, intraperitoneal administration and intravenous administration, and to examine the potential applications of miR-145 in ovarian cancer therapeutics.

\section{Acknowledgements}

Not applicable.

\section{Funding}

The present study was supported by The Science Plan Program of Nantong Municipal Health and Family Planning Commission (grant no. WQ2016073), The Science Plan Program of Nantong (grant no. MS12017014-5), Jiangsu Provincial Key Medical Talents Program for Youth (grant no. QNRC2016404) and The Six-One Project of Jiangsu Provincial Health Committee (grant no. LGY2018036).

\section{Availability of data and materials}

All data generated or analyzed during the present study are included in this published article.

\section{Authors' contributions}

MH and JZ designed the experiments. YQ, MS, JY and YC performed the experiments. $\mathrm{XC}$ and $\mathrm{WC}$ analyzed the data. $\mathrm{MH}$ and $\mathrm{JZ}$ interpreted the data. YQ wrote the manuscript. All authors read and approved the manuscript.

\section{Ethics approval and consent to participate}

The present study was approved by The Ethics Committee of the Affiliated Hospital of Nantong University. Written informed consent was obtained from all enrolled subjects.

\section{Patient consent for publication}

All patients within this study provided consent for the publication of their data.

\section{Competing interests}

The authors declare that they have no competing interests.

\section{References}

1. Novoa-Vargas A: Natural history of ovary cancer. Ginecol Obstet Mex 82: 613-622, 2014 (In Spanish).

2. Bartel DP: MicroRNAs: Genomics, biogenesis, mechanism, and function. Cell 116: 281-297, 2004.

3. Garofalo $\mathrm{M}$ and Croce CM: microRNAs: Master regulators as potential therapeutics in cancer. Annu Rev Pharmacol Toxicol 51: 25-43, 2011.

4. Mendell JT and Olson EN: MicroRNAs in stress signaling and human disease. Cell 148: 1172-1187, 2012.

5. Iorio MV, Visone R, Di Leva G, Donati V, Petrocca F, Casalini P, Taccioli C, Volinia S, Liu CG, Alder H, et al: MicroRNA signatures in human ovarian cancer. Cancer Res 67: 8699-8707, 2007.

6. Zhang J, Guo H, Zhang H, Wang H, Qian G, Fan X, Hoffman AR, $\mathrm{Hu}$ JF and Ge S: Putative tumor suppressor miR-145 inhibits colon cancer cell growth by targeting oncogene Friend leukemia virus integration 1 gene. Cancer 117: 86-95, 2011.

7. Zhu Z, Xu T, Wang L, Wang X, Zhong S, Xu C and Shen Z: MicroRNA-145 directly targets the insulin-like growth factor receptor I in human bladder cancer cells. FEBS Lett 588: 3180-3185, 2014

8. Sachdeva M, Zhu S, Wu F, Wu H, Walia V, Kumar S, Elble R, Watabe K and Mo YY: p53 represses c-Myc through induction of the tumor suppressor miR-145. Proc Natl Acad Sci USA 106: 3207-3212, 2009.

9. Zhang J, Guo H, Qian G, Ge S, Ji H, Hu X and Chen W: MiR-145, a new regulator of the DNA fragmentation factor- 45 (DFF45)-mediated apoptotic network. Mol Cancer 9: 211, 2010.

10. Sachdeva M and Mo YY: MicroRNA-145 suppresses cell invasion and metastasis by directly targeting mucin 1 . Cancer Res 70 : 378-387, 2010.

11. Xu Q, Liu LZ, Qian X, Chen Q, Jiang Y, Li D, Lai L and Jiang BH: MiR-145 directly targets p70S6K1 in cancer cells to inhibit tumor growth and angiogenesis. Nucleic Acids Res 40: 761-774, 2012.

12. Cho WC, Chow AS and Au JS: MiR-145 inhibits cell proliferation of human lung adenocarcinoma by targeting EGFR and NUDT1. RNA Biol 8: 125-131, 2011.

13. Chan M, Liaw CS, Ji SM, Tan HH, Wong CY, Thike AA, Tan PH, Ho GH and Lee AS: Identification of circulating microRNA signatures for breast cancer detection. Clin Cancer Res 19: 4477-4487, 2013 
14. Gramantieri L, Fornari F, Ferracin M, Veronese A, Sabbioni S, Calin GA, Grazi GL, Croce CM, Bolondi L and Negrini M: MicroRNA-221 targets Bmf in hepatocellular carcinoma and correlates with tumor multifocality. Clin Cancer Res 15: 5073-5081, 2009.

15. Ichimi T, Enokida H, Okuno Y, Kunimoto R, Chiyomaru T, Kawamoto K, Kawahara K, Toki K, Kawakami K, Nishiyama K, et al: Identification of novel microRNA targets based on microRNA signatures in bladder cancer. Int J Cancer 125: 345-352, 2009.

16. Amaral FC, Torres N, Saggioro F, Neder L, Machado HR, Silva WJ Jr, Moreira AC and Castro M: MicroRNAs differentially expressed in ACTH-secreting pituitary tumors. J Clin Endocrinol Metab 94: 320-323, 2009.

17. Yang D, Sun Y,Hu L, Zheng H, Ji P, Pecot CV, Zhao Y, Reynolds S, Cheng H, Rupaimoole R, et al: Integrated analyses identify a master microRNA regulatory network for the mesenchymal subtype in serous ovarian cancer. Cancer Cell 23: 186-199, 2013.

18. Chung YW, Bae HS, Song JY, Lee JK, Lee NW, Kim T and Lee KW: Detection of microRNA as novel biomarkers of epithelial ovarian cancer from the serum of ovarian cancer patients. Int J Gynecol Cancer 23: 673-679, 2013.

19. Letelier P, García P, Leal P, Álvarez H, Ili C, López J, Castillo J, Brebi P and Roa JC: miR-1 and miR-145 act as tumor suppressor microRNAs in gallbladder cancer. Int J Clin Exp Pathol 7: 1849-1867, 2014

20. Wu H, Xiao Z, Wang K, Liu W and Hao Q: MiR-145 is downregulated in human ovarian cancer and modulates cell growth and invasion by targeting p70S6K1 and MUC1. Biochem Biophys Res Commun 441: 693-700, 2013.

21. Zhang W, Wang Q, Yu M, Wu N and Wang H: MicroRNA-145 function as a cell growth repressor by directly targeting c-Myc in human ovarian cancer. Technol Cancer Res Treat 13: 161-168, 2014.

22. Zhang Q, Sakamoto K and Wagner KU: D-type Cyclins are important downstream effectors of cytokine signaling that regulate the proliferation of normal and neoplastic mammary epithelial cells. Mol Cell Endocrinol 382: 583-592, 2014.

23. Kato JY and Sherr CJ: Inhibition of granulocyte differentiation by G1 cyclins D2 and D3 but not D1. Proc Natl Acad Sci USA 90: 11513-11517, 1993.

24. Song H, Hogdall E, Ramus SJ, Dicioccio RA, Hogdall C, Quaye L, McGuire V, Whittemore AS, Shah M, Greenberg D, et al: Effects of common germ-line genetic variation in cell cycle genes on ovarian cancer survival. Clin Cancer Res 14: 1090-1095, 2008.

25. Rady B, Chen Y, Vaca P, Wang Q, Wang Y, Salmon P and Oberholzer J: Overexpression of E2F3 promotes proliferation of functional human $\beta$ cells without induction of apoptosis. Cell Cycle 12: 2691-2702, 2013.

26. Bracken AP, Ciro M, Cocito A and Helin K: E2F target genes: Unraveling the biology. Trends Biochem Sci 29: 409-417, 2004.

27. Johnson DG, Schwarz JK, Cress WD and Nevins JR: Expression of transcription factor E2F1 induces quiescent cells to enter S phase. Nature 365: 349-352, 1993.

28. Nevins JR: E2F: A link between the Rb tumor suppressor protein and viral oncoproteins. Science 258: 424-429, 1992.

29. Blais A and Dynlacht BD: Hitting their targets: An emerging picture of E2F and cell cycle control. Curr Opin Genet Dev 14 527-532, 2004

30. Livak KJ and Schmittgen TD: Analysis of relative gene expression data using real-time quantitative PCR and the 2(-Delta Delta C(T)) method. Methods 25: 402-408, 2001.
31. Zhu H, Dougherty U, Robinson V, Mustafi R, Pekow J, Kupfer S, Li YC, Hart J, Goss K, Fichera A, et al: EGFR signals downregulate tumor suppressors miR-143 and miR-145 in Western diet-promoted murine colon cancer: Role of G1 regulators. Mol Cancer Res 9: 960-975, 2011

32. Le Beau MM, Lemons RS, Espinosa R III, Larson RA, Arai N and Rowley JD: Interleukin-4 and interleukin-5 map to human chromosome 5 in a region encoding growth factors and receptors and are deleted in myeloid leukemias with a del(5q). Blood 73: 647-650, 1989

33. Zhu X, Li Y, Xie C, Yin X, Liu Y, Cao Y, Fang Y, Lin X, Xu Y, $\mathrm{Xu}$ W, et al: miR-145 sensitizes ovarian cancer cells to paclitaxel by targeting Sp1 and Cdk6. Int J Cancer 135: 1286-1296, 2014.

34. Gadducci A, Sergiampietri C, Lanfredini N and Guiggi I: Micro-RNAs and ovarian cancer: The state of art and perspectives of clinical research. Gynecol Endocrinol 30: 266-271, 2014.

35. Shi B, Sepp-Lorenzino L, Prisco M, Linsley P, DeAngelis T and Baserga R: Micro RNA 145 targets the insulin receptor substrate-1 and inhibits the growth of colon cancer cells. J Biol Chem 282: 32582-32590, 2007

36. La Rocca G, Badin M, Shi B, Xu SQ, Deangelis T, Sepp-Lorenzinoi L and Baserga R: Mechanism of growth inhibition by MicroRNA 145: The role of the IGF-I receptor signaling pathway. J Cell Physiol 220: 485-491, 2009.

37. Fuse M, Nohata N, Kojima S, Sakamoto S, Chiyomaru T, Kawakami K, Enokida H, Nakagawa M, Naya Y, Ichikawa T and Seki N: Restoration of miR-145 expression suppresses cell proliferation, migration and invasion in prostate cancer by targeting FSCN1. Int J Oncol 38: 1093-1101, 2011.

38. Speranza MC, Frattini V, Pisati F, Kapetis D, Porrati P, Eoli M, Pellegatta S and Finocchiaro G: NEDD9, a novel target of miR-145, increases the invasiveness of glioblastoma. Oncotarget 3: 723-734, 2012

39. Yu CC, Tsai LL, Wang ML, Yu CH, Lo WL, Chang YC, Chiou GY, Chou MY and Chiou SH: miR145 targets the SOX9/ADAM17 axis to inhibit tumor-initiating cells and IL-6-mediated paracrine effects in head and neck cancer. Cancer Res 73: 3425-3440, 2013.

40. Sherr CJ: G1 phase progression: Cycling on cue. Cell 79: 551-555, 1994.

41. Weinberg RA: The retinoblastoma protein and cell cycle control. Cell 81: 323-330, 1995.

42. Trimarchi JM and Lees JA: Sibling rivalry in the E2F family. Nat Rev Mol Cell Biol 3: 11-20, 2002

43. He Y, Franco OE, Jiang M, Williams K, Love HD, Coleman IM, Nelson PS and Hayward SW: Tissue-specific consequences of cyclin D1 overexpression in prostate cancer progression. Cancer Res 67: 8188-8197, 2007.

44. Chang L, Guo R, Yuan Z, Shi H and Zhang D: LncRNA HOTAIR regulates CCND1 and CCND2 expression by sponging miR-206 in ovarian cancer. Cell Physiol Biochem 49: 1289-1303, 2018.

45. Reimer D, Hubalek M, Riedle S, Skvortsov S, Erdel M, Concin N, Fiegl H, Müller-Holzner E, Marth C, Illmensee $\mathrm{K}$, et al: E2F3a is critically involved in epidermal growth factor receptor-directed proliferation in ovarian cancer. Cancer Res 70: 4613-4623, 2010.

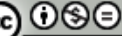

This work is licensed under a Creative Commons Attribution-NonCommercial-NoDerivatives 4.0 International (CC BY-NC-ND 4.0) License. 